\title{
HERNIAS POCO COMUNES: HERNIA DE SPIEGEL. REPORTE DE CASO
}

\author{
MARIANA AÑOLIS' 10 \\ ANDRÉS REYES ${ }^{\prime}$ \\ ADEL AL AWAD $1,2,3$ \\ MARÍA MATERA ${ }^{1,4,5}$
}

\section{UNCOMMON HERNIAS: SPIGELIAN HERNIA. CASE REPORT}

\begin{abstract}
RESUMEN
La hernia de Spiegel es un defecto infrecuente de la pared abdominal producido por una alteración en la unión de borde lateral del músculo recto del abdomen y el borde medial del músculo transverso del abdomen en la línea semilunar. Representan apenas del 0,12 al 2\% de todas las hernias. La presente investigación se enfocó en describir las manifestaciones clínicas, el diagnóstico, la incidencia, el procedimiento quirúrgico y su evolución en dos casos reportados. Caso 1: Femenina de 33 años, multípara, obesa, quien consulta por dolor y masa palpable en el cuadrante superior izquierdo del abdomen. Al examen físico, se evidenció dolor a la palpación en mesogastrio e hipocondrio izquierdo, exhibiendo protrusión del contenido abdominal, en la línea de Spiegel. Intervención quirúrgica: hernioplastia protésica de Spiegel con colocación de malla supraaponeurótica. Caso 2: Masculino de 89 años, con diagnóstico de EPOC, acudió a consulta presentando dolor abdominal en fosa iliaca y en la región inguino-escrotal izquierda. Al examen físico se evidenció saco herniario en la región inguino-escrotal por lo que se decide su resolución quirúrgica. Como hallazgo transoperatorio se evidenció defecto aponeurótico del recto anterior del abdomen izquierdo en su tercio inferior. Intervención quirúrgica: hernioplastia protésica de Spiegel con colocación de malla supraaponeurótica. Conclusión: La hernia de Spiegel es una entidad poco frecuente por lo que se debe concientizar al cirujano para sospechar este tipo de hernia en aquellos pacientes con presencia de aumento de volumen en la línea de Spiegel.
\end{abstract}

Palabras clave: Reporte de casos, hernia de Spiegel, hernioplastia, malla supraaponeurótica.

1. CEDIAH: Comunidad Estudiantil para la Difusión e Investigación de la Anatomía Humana. Escuela de Medicina. Universidad del Zulia, Maracaibo, Venezuela. Correo-e: mariananolis16@ gmail.com

2. Cirujano hepatobiliopancreático del Hospital Coromoto de Maracaibo.

3. Director del curso deampliación de Cirugía Hepatobiliopancreático Hospital Coromoto de Maracaibo

4. Docente libre de la Cátedra de Anatomía Humana. Facultad de Medicina. Universidad del Zulia, Maracaibo, Venezuela

5. Adjunta del Servicio de Cirugía General. Hospital Universitario de Maracaibo, Venezuela.

\section{ABSTRACT}

Spigelian hernia is an infrequent abdominal wall defect caused by an alteration in the joint between lateral edge of the rectus abdominis muscle and the medial egde of the transverse abdominis muscle in the semilunar line. It represent only from 0.12 to $2 \%$ of all hernias. This investigation is focussed on describe the clinical manifestations, diagnosis, incidence, the surgical procedure and its evolution in two reported cases. Case 1: A 33 years old female, multiparous, obese, who consulted for pain and palpable mass in the left upper quadrant of the abdomen. Physical examination revealed tenderness between mesogastrium and left hypochondrium, showing protrusion of abdominal content, in the Spigelian line. Surgical intervention: Prosthetic Spiegel hernioplasty with supraaponeurotic mesh. Case 2: An 89 years old male, diagnosed with COPD, attended in the consultation presenting abdominal pain in the iliac fossa and in the left inguino-scrotal region. Physical examination revealed hernial sac in the inguino-scrotal region, reason which its surgical resolution is decided. As an intraoperative finding, an aponeurotic defect of the anterior rectus of the left abdomen was evidenced in its lower third. Surgical intervention: Prosthetic Spiegel hernioplasty with supraaponeurotic mesh. Conclusion: Spigelian hernia is a rare entity, so the surgeon must be focused on suspect this kind of hernia in patients with growth in Spiegel line.

Key words: Cases report, Spigelian hernia, hernioplasty, supraaponeuotic mesh

DOI: $\underline{10.48104 / R V C .2020 .73 .2 .9}$ 


\section{INTRODUCCIÓN}

La hernia de Spiegel es un defecto infrecuente de la pared abdominal producido por una alteración en la unión del borde lateral del músculo recto del abdomen y el borde medial del músculo transverso del abdomen en la línea semilunar o línea de Spiegel. ." Suelen aparecer en el "Cinturón de Spiegel", una franja imaginaria que se extiende $6 \mathrm{~cm}$ por encima de las espinas ilíacas antero-superiores. ${ }^{(2)}$ Asimismo, representan apenas del 0, 12 al 2\% de las hernias de la pared abdominal. (1,3) (Figura 1)

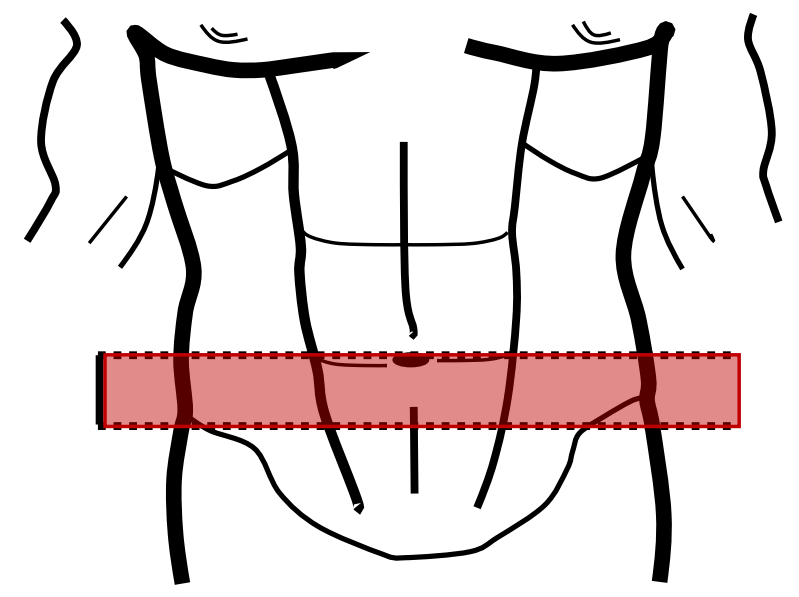

Figura 1. En color rojo se delimita el cinturón de Spiegel

Existen varios factores que contribuyen a su etiología, se describen aquellos que producen aumento de la presión intraabdominal. ${ }^{(4)}$ Comúnmente se presenta con dolor abdominal, sensación de masa y/o aumento de volumen en la región abdominal." "' La presente investigación tiene como objetivo describir las manifestaciones clínicas, el diagnóstico, la incidencia, el procedimiento quirúrgico, además de la evolución en los dos casos reportados con hernia de Spiegel

\section{Presentación de los casos \\ REPORTE DE CASO 1 \\ Información del Paciente}

Paciente femenina de 33 años de edad, multípara, con diagnóstico de obesidad, antecedentes de esófago de Barret y gastritis erosiva asociada a $\boldsymbol{H}$. py lori. Presentó además antecedente de cesárea anterior, negando otras cirugías previas. No presentó antecedentes familiares de importancia.

\section{Hallazgos Clínicos}

Consultó al servicio de emergencia por presencia de dolor abdominal de inicio insidioso, de fuerte intensidad, en mesogastrio e hipocondrio izquierdo, que se agravaba con el movimiento y no mejoraba con la administración de analgésicos tipo antiinflamatorio no esteroideos (AINEs). Aunado al cuadro, manifestó aumento de volumen abdominal entre epigastrio e hipocondrio izquierdo. Al examen físico, se evidenció dolor a la palpación en mesogastrio e hipocondrio izquierdo, exhibiendo protrusión del contenido abdominal entre el epigastrio y el hipocondrio izquierdo, inmóvil, además de defecto de la pared de $5 \mathrm{~cm}$ en la línea de Spiegel. Motivo por el cual ingresó con diagnóstico preoperatorio de hernia de Spiegel.

\section{Estudios diagnósticos}

Los exámenes de laboratorio no reflejaron alteraciones. No se realizaron estudios diagnósticos de imágenes debido a que el paciente no contaba con los recursos económicos para su realización.

\section{Tratamiento realizado}

Tras el diagnóstico, se realizaron los exámenes preoperatorios pertinentes y la cirugía para su resolución mediante el abordaje abierto. Con la paciente en decúbito supino, y bajo anestesia general, se efectuó incisión longitudinal para-rectal izquierda, con disección por plano la cual arrojó como hallazgo la presencia de defecto aponeurótico de $5 \mathrm{~cm}$ aproximadamente en el borde externo del músculo recto anterior del abdomen izquierdo en su tercio superior. Luego, se verificó la debilidad y se disecó el saco herniario en el cuál se evidenció la presencia de un segmento de asa delgada. (Figura 2) Se procedió a la reducción del contenido y cierre de la aponeurosis para posteriormente realización de hernioplastia protésica de Spiegel con colocación de malla supraaponeurótica. El cierre se realizó con poliglactina 9101.

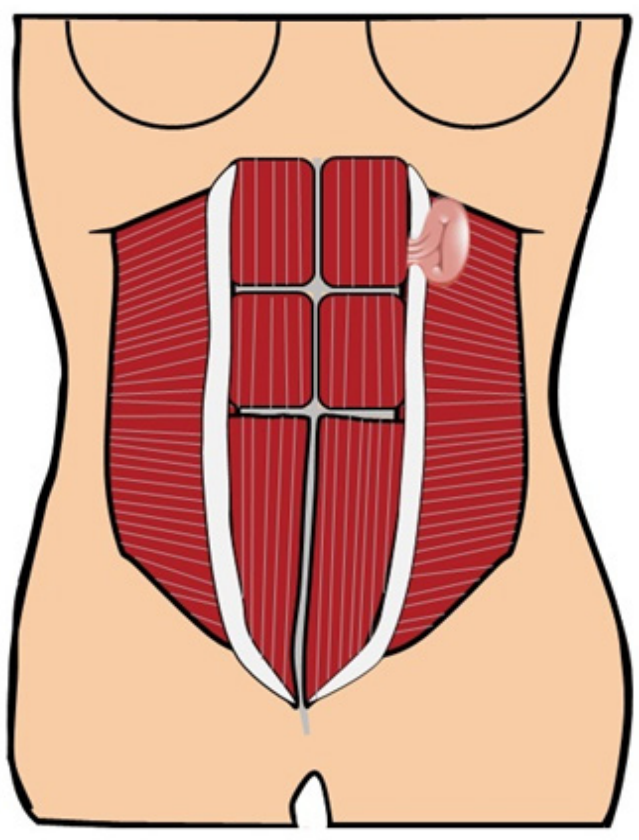

Figura 2. Localización topográfica del saco herniario con su contenido 


\section{Seguimiento y resultados}

Ulterior a la intervención, el periodo postoperatorio de la paciente cursó sin eventualidades, por consecuente, en las siguientes $24 \mathrm{~h}$ al acto quirúrgico, se decidió el alta médica. Se dió seguimiento por consulta externa durante 6 meses donde no se presentaron recidivas.

\section{REPORTE DE CASO 2}

\section{Información del paciente}

Paciente masculino, caucásico de 89 años de edad, como antecedente resaltante de ser fumador, con diagnóstico de enfermedad pulmonar obstructiva crónica (EPOC) desde hace 10 años, hipertensión arterial datado desde hace 20 años, sin antecedentes familiares de importancia ni cirugías previas.

\section{Hallazgos clínicos}

Acudió a la consulta presentando dolor abdominal insidioso, de moderada intensidad, localizado en fosa iliaca izquierda y en la región inguino - escrotal ipsilateral, de 3 meses de evolución, que se exacerbaba con el movimiento y la tos, el cual no mitigaba con la administración de AINEs. Durante la exploración física se evidenció saco herniario en la región inguino - escrotal, además de dolor a la palpación en fosa iliaca izquierda, protrusión del contenido abdominal, móvil, con defecto de más de $5 \mathrm{~cm}$. Se ingresó con el diagnóstico preoperatorio de hernia inguinoescrotal.

\section{Estudios diagnósticos}

Los exámenes de laboratorio no presentaron alteraciones No se realizaron imágenes diagnósticas al no contar el paciente con los recursos económicos para su ejecución.

\section{Tratamiento realizado}

Tras obtener la evaluación cardiovascular correspondiente y los exámenes de laboratorio preoperatorios, bajo anestesia general y con el paciente en decúbito supino, se realizó la intervención mediante el abordaje abierto para el tratamiento de la hernia inguino-escrotal. Durante la misma, se evidenció un segundo defecto aponeurótico en el extremo lateral del músculo recto anterior del abdomen izquierdo en su tercio inferior. Por lo que se agregó el diagnóstico de Hernia de Spiegel. Para su resolución, se efectuó incisión longitudinal pararrectal izquierda, Posteriormente se realizó la disección por plano y verificación de la debilidad, disección del saco herniario y reducción de contenido el cual correspondía a epiplón. (Figura 3) Luego del cierre de la aponeurosis se realizó hernioplastia protésica de Spiegel con colocación de malla supraaponeurótica.

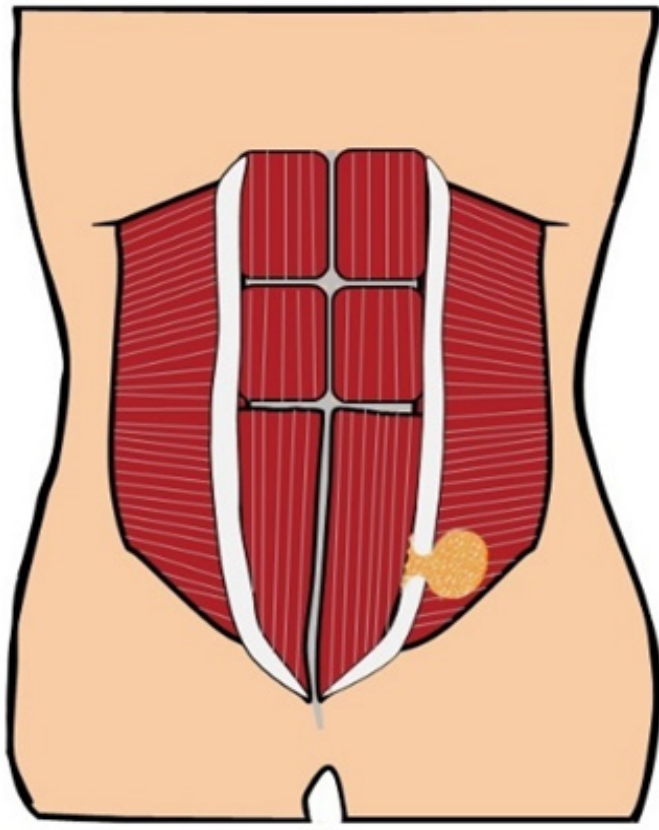

Figura 3. Representación gráfica del sitio donde se presento el defecto, observándose el saco herniario y su contenido

\section{Seguimiento y resultados}

El periodo postoperatorio transcurrió de manera favorable y el paciente egresó al tercer día posterior a la cirugía. Se evaluó la evolución durante un periodo de 6 meses, sin evidencia clínica de recidiva ni complicaciones.

\section{DISCUSIÓN}

La hernia de Spiegel es una entidad poco frecuente. (1) El 85 - 95\% de las hernias de Spiegel se producen en una franja de $6 \mathrm{~cm}$ por encima de ambas espinas iliacas antero-superiores, sin embargo, también puede presentarse en su límite superior ${ }^{15}$ Además, estas suelen presentarse en el lado izquierdo con mayor frecuencia..$^{(4)}$ Su diagnóstico clínico puede ser desafiante debido a sus manifestaciones clínicas inespecíficas y al amplio abanico de diagnósticos diferenciales. ${ }^{(6)}$

Respecto a su incidencia, representan menos del 2\% de las hernias de la pared abdominal. ${ }^{(7)}$ La relación en la aparición de la hernia de Spiegel en cuanto al sexo corresponde a 1.7:1 siendo más común en mujeres. (8) Por otra parte, su pico de incidencia ocurre alrededor de los 60 años de edad, aunque puede presentarse en cualquier edad. ${ }^{(9)}$ Existen factores que contribuyen en su etiología, agrupando a aquellos que producen aumento de la presión intraabdominal como la obesidad, multiparidad, ascitis, cirugías previas, EPOC y traumatismos. ${ }^{(4,8)}$

El 50\% de las hernias de Spiegel son diagnosticadas en el acto operatorio. ${ }^{(3)}$ Su tratamiento es quirúrgico, mediante las 
técnicas de herniorrafía o hernioplastia por vía convencional o laparoscópica.(5) El abordaje común para reparar este defecto es a través de la cirugía abierta, sin embargo, el abordaje laparoscópico está reportado en numerosos casos.(3,10) Un estudio aleatorizado prospectivo comparó pacientes sometidos a una reparación abierta vs aquellos a quienes se les realizó el abordaje laparoscópico, estos últimos mostraron ventaja con respecto a la morbilidad. (11)

El diagnóstico de la hernia de Spiegel puede realizarse de forma preoperatoria y transoperatoria. Para su resolución, el abordaje convencional mediante la hernioplastia protésica con colocación de malla supraaponeurótica arroja resultados satisfactorios tanto en la recuperación como en la evolución de los pacientes.

Finalmente, al ser una patología poco frecuente, se debe concientizar al cirujano para sospechar este tipo de hernia en pacientes con presencia de aumento de volumen en la línea de Spiegel, especialmente en aquellos que presentan comorbilidades. Además, recomendamos realizar una adecuada exploración física aunado a un interrogatorio exhaustivo para así facilitar el diagnóstico, reducir costos de estudios diagnósticos y disminuir la incidencia de sus complicaciones.

\section{REFERENCIAS}

1. Dilzib EA, Ortiz SF, Morales JI, Nuñez R, Rodarte G, Leal G. Reparación laparoscópica de hernia de Spiegel con colocación de malla compuesta intraperitoneal y sistema de fijación articulado. Reporte de dos casos. Cir. Cir. 2019; 87(3):253-257.

2. Lasarte AS, Ortega HM, de la Torre MN, Gutierrez O, García A, Aguirre $L$, et al. Apendicitis aguda por hernia de Spiegel incarcerada. Rev. Hispanoam. Hernia. 2014; 2(3):115-118.

3. Socea B, Smaranda A, Nica A, et al. Spiegel hernia-case presentation. Arch Balk Med Union. 2018; 53(1):152-154.

4. Moreno A, Flores B, Aguayoa J, Canteras M. La hernia de Spiegel en España: revisión bibliográfica y presentación de una serie personal de 27 pacientes. Cir. Esp. 2002; 72(1):18-22.

5. Rodriguez J, Ramírez J, Rodríguez J, Hernández M. Spigelian hernia: case report. Gac. Med. Espirit. 2019; 21(2):121-127.

6. Foster D, Nagarajan S, Panait L. Richter-type Spigelian hernia: A case report and review of the literature. Int J Surg Case Rep. 2015; (6): 160-162.

7. Mederos R, Lamas JR, Alvarado J, Matos M, Padron I, Ramos A. Laparoscopic diagnosis and repair of Spigelian hernia: a case report and literature review. Int J Surg Case Rep. 2017; 31:184-187.

8. Moles L, Docobo F, Mena J, Quinta R. Hernia de Spiegel en España: Análisis de 162 casos. Rev. Esp. Enferm. Dig. 2005; 97(5):338-347.

9. Ussia A, Imperato F, Schindler L, Wattiez A, Koninckx PR. Spigelian hernia in gynaecology. Gynecol Surg. 2017; 14(1): 1-4.
10. Matsui S, Nitori N, Kato A, Ikeda Y, Kiatagwa Y, Hasegawa $H$, et al. Laparoscopic totally extra-peritoneal hernia repair for bilateral Spigelian hernias and coincident inguinal hernia: A case report. Int J Surg Case Rep. 2016; 28:169-172

11. Moreno-Egea A, Carrasco L, Girela E, Martin JG, Aguayo JL, Canteras M. Open vs laparoscopic repair of spigelian hernia a prospective randomized trial. Arch Surg. 2002; 137:1266-1268 